\title{
Chronic pelvic pain
}

\author{
Slawomir Wozniak ${ }^{1}$ \\ 1 3rd Department of Gynecology, Medical University, Lublin, Poland \\ Wozniak S. Chronic pelvic pain. Ann Agric Environ Med. 2016; 23(2): 223-226. doi: 10.5604/12321966.1203880
}

\begin{abstract}
Introduction. Chronic pelvic pain (CPP) affects about $10-40 \%$ of women presenting to a physician, and is characterised by pain within the minor pelvis persisting for over 6 months.

Materials and method. The Medline database was searched using the key words 'chronic pelvic pain' and 'pelvic congestion syndrome', published in English during the past 15 years.

The condition markedly deteriorates the quality of life of the affected. Its aetiology has not been fully described and elucidated, although organic, functional and psychosomatic factors are implicated. Pain associated with parametrial varices was defined as pelvis congestion syndrome (PCS). Since the aetiology of CPP is complex, multi-directional diagnostic procedures are required.

Results. The main diagnostic methods employed are imaging examinations (ultrasound, computer tomography, magnetic resonance). Advances in interventional radiology considerably contributed to the CPP treatment. Currently, embolization of parametrial vessels is one of the most effective methods to relieve pain associated with pelvic congestion syndrome. Conclusions. Due to the complex aetiology of chronic pelvic pain, the most beneficial effects are obtained when the therapy is based on cooperation of the gynaecologist, physiotherapist, psychologist and interventional radiologist.
\end{abstract}

\section{Key words}

chronic pelvic pain, pelvic congestion syndrome, parametrial varices

\section{INTRODUCTION}

Chronic Pelvic Pain (CPP) is defined as continuous or intermittent pain located in the hypogastrium (minor pelvis) persisting for more than 6 months. CPP is a widespread condition affecting about 1 in 6 adult females [1]. Increased pain deteriorates the quality of life of the patients and requires pharmacological treatment or surgical interventions. It is estimated that about $3.8 \%$ of women at any age and $12 \%$ of those at reproductive age complain of chronic pain sensations in the minor pelvis region [2]. Data from the United Kingdom reveal that about $18 \%$ of women take one day of sick leave annually due to CPP. The findings reported by Perry et al. indicate that about $2-10 \%$ of women presenting to the Gynaecological Outpatient Department suffer from CPP, of whom about $20 \%$ undergo diagnostic laparoscopy. According to Gelbay et al., $10 \%-15 \%$ of CPP patients are qualified for a hysterectomy $[3,4,5]$.

The aetiology of CPP has not been fully defined and elucidated. The causes are usually complex. Several paininducing factors are implicated. Acute pain is caused by tissue damage and decreases or subsides simultaneously with healing. Since the aetiology of chronic pain is affected by some additional factors, it can persist long after the tissue injury has healed or occur despite its absence. The lesions involve both the ascending and descending tracts of the central and peripheral nervous system. The immunological factors inducing peripheral nervous system dysfunction and activating normally inactive fibres, including cytokines, such as tumour necrosis factor $a($ TNF- $\alpha)$ and chemokines.

The above-mentioned locally secreted factors cause dysaesthesia involving a larger area than primarily affected by

Address for correspondence: Slawomir Wozniak, 3rd Department of Gynecology Medical University, Lublin, Poland

E-mail: slavw07572@gmail.com

Received: 17 April 2014; accepted: 18 July 2014 the pathology. Long-term pain can also enhance the primary pain stimulus, which leads to visceral hyperalgesia. Surgeryrelated damage to nervous fibres, injury, or inflammation, can induce or increase pain sensations $[5,6]$. According to the Royal College of Obstetricians and Gynaecologists (RCOG), the aetiological factors causing pain can be divided into those that are gynaecological and extra-gynaecological [7]. The gynaecological factors causing CPP include:

- endometriosis / adenomyosis

- pelvic congestion syndrome

- uterine fibroids

- ovarian tumours

- pelvic inflammatory disease

- post-operative or post-inflammatory adhesions.

The extra-gynaecological factors causing CPP are as follows:

- surgical (chronic appendicitis, adhesions)

- urological (interstitial cystitis, chronic urinary inflammation, urolithiasis, urethra syndrome)

- gastrointestinal (irritable bowel syndrome, constipations, inflammatory bowel diseases)

- ortho-neuro-muscular (degenerative changes, neuropathies, prolapse of the nucleus pulposus, nerve incarceration)

- psychosomatic (depression, sleep disorders, anxiety, migraine with abdominal symptoms, history of sexual abuse)

- neurological (nerve incarceration in the scar inducing chronic pain in the region supplied by the affected nerve; in about $3.7 \%$ of cases it is caused by a single Pfannenstiel incision).

Endometriosis and adenomyosis are common causes of CPP. According to Neis, endometriosis is diagnosed in about $1 / 3$ of women with CPP and in less than $5 \%$ of those without CPP [8]. 
Endometriosis develops primarily in reproductive age women, between 15-49 years of age, yet may also be diagnosed during puberty and after menopause. Typical symptoms of endometriosis, as well as adenomyosis, include dysmenorrhoea, dyspareunia, and chronic pelvic pain [2]. The severity of symptoms depends on the stage of endometriosis according to the American Society for Reproductive Medicine Classification for Endometriosis (ASRM), size and number of endometriosis lesions, involvement of the Douglas sinus and number of surgical interventions in the pelvic region [9]. Randal et al. demonstrated that women complaining of CPP and with suspected endometriosis had significantly higher levels of antiendometrial antibodies (AEAs), regulatory $\mathrm{T}$ lymphocytes (Tregs), nerve growth factor (NGF) in the area affected by endometriosis and vascular endothelial growth factor (VEGF) [10]. The immune response is modulated, among other factors, by increased levels of estrogens, which is observed in patients with endometriosis. According to the literature data, these mechanisms are likely to underlie CPP causing the ingrowth of nerve fibres to the endometrial ectopic foci and neoangiogenesis in nerves. This mechanism is implicated in the enhancement of pain sensations. Moreover, available reviews regarding this issue indicate that pain is attributable to increased inflammation. The literature findings demonstrate that women with endometriosis have higher levels of inflammatory factors in the peritoneal fluid and blood serum $[2,11,12]$. Significantly higher levels of leptin and 6-keto-prostaglandin F1 alpha (6-KF) were found in the peritoneal fluid in women with endometriosis [13, 14, 15]. According to Drosdzol-Cop et al., adolescent girls diagnosed with endometriosis have statistically significantly higher serum levels of interleukin 4 (IL-4). Moreover, significantly decreased levels of IL-2 and increased levels of IL-6, tumour necrosis factor $-\alpha$ (TNF- $\alpha$ ) and glycodelin A, were noted in the study group [16].

Pelvic congestion syndrome (PCS) is believed to be one of the causes of CPP. The aetiology of PCS has not been fully explained. The theory regarding the pain due to dilation of venous vessels and reduced flow in pelvic vessels was first put forward by Taylor in 1949 [17]. One of PCS factors is likely to be failure or lack of the valve system in the peri-ovarian and parametrial veins. The anomaly of vessels causes retrograde flow to the ovarian vessels, which results in visibly dilated veins and varices. The lack of valves near the ovarian vessel ramification is found in about $15 \%$ of women; the failure of the valve system is diagnosed in $40 \%$ of cases on the left side and in $35 \%$ of patients on the right side $[18,19]$. Mechanical vessel compression impairs blood flow, e.g. improper position of the uterus or shift of the uterus can cause the compression of the left renal vein between the aorta and the inferior mesenteric artery. Veins can increase their volume by about $60 \%$ and therefore symptoms are initially less distinct $[20,21]$.

Moreover, $15 \%$ of women aged $20-50$ are diagnosed with pelvic varices, which are not always symptomatic. The risk factors of pelvic congestion syndrome include the number of pregnancies $>=2$, varices of the lower limbs, polycystic ovary syndrome and hormonal disorders. Increased levels of estrogens correlate with the development of PCS. By weakening the vascular walls, estrogens may induce varices. The incidence of PCS is found to be higher in women before menopause. Hormonal changes occurring during pregnancy and the enlarging uterus increase the volume of pelvic veins by even $60 \%$, slowing down the blood flow, causing the mass effect and compressing the adjacent nerves, which results in pain sensations. Moreover, the uterus may also compress vessels, which may lead to the development of pain and PCS $[19,20,21]$.

PCS is characterised by a chronic, dull, continuous pain, which often increases after intercourse, before or during the first days of menstruation, in the standing and sitting position, during pregnancy, and in cases of irregular menstruation and perineal varices. In the majority of cases, pain occurs on the side of venostasis, less frequently on the opposite side. PCS can also be accompanied by urinary symptoms caused by perineal varices, lumbosacral pain, nausea, flatulence, cramping abdominal pains. The physical examination reveals varices along the saphenous vein and pain sensations in the ovarian projection.

Pelvic Doppler ultrasound is the first-line imaging examination used for PCS diagnosis. In cases of PCS, the examination shows enhanced flows within the minor pelvis $[20,21]$. Park et al. visualised pelvic minor varices in 53\% of the female population [22]. In the majority of centres, computer tomography with contrast has replaced venography for imaging vessels of the minor pelvis. An alternative method without contrast is magnetic resonance imaging (MRI). MRI visualises the dilated, tortuous vessels in the area of the ovary and uterus, which can reach the broad ligament of the pelvis or the pelvic wall. The use of gadolinium increases the sensitivity of this examination by mapping the vessels of the minor, and precisely localises the pathology [21].

Laparoscopy used for the diagnosis of CPP may not visualise the dilated vessels. Increased abdominal pressure can compress the vessels and mask the presence of pathologically dilated vessels. The results of diagnostic laparoscopy in PCS are normal in $80-90 \%$ of cases [21]. However, in certain cases, laparoscopy enables visualisation of the causes of PCS, e.g. foci of endometriosis.

The treatment of PCS is mainly dependent on the severity of pain. Symptomatically acting analgesics, i.e. nonsteroidal anti-inflammatory drugs, are used. In cases of pain associated primarily with menstruation, contraceptive pills are used to reduce the heaviness of menstruation or as continuous therapy to inhibit menstruation. Moreover, the use of medroxyprogesterone for the treatment of PCS has been described in some prospective studies. Thanks to its vasoconstricting properties, six-month therapy alleviated pain sensations and suppressed symptoms reported by patients. However, the effect was not long-lasting. Pain has also been treated with gonadotropin-releasing hormone (GnRH) agonists with hormones used for hormone replacement therapy (HRT), dihydroergotamine, goserelin, danazol, and substances reconstructing the vascular wall [20, 23-26].

Once pharmacological treatment options have failed, invasive methods are applied. Prior to the development of interventional radiology, hysterectomy with salpingectomy or ovariectomy was performed. At present, one of the most common methods providing satisfactory outcomes is embolization. The procedure was first used for the treatment of PCS by Edwards et al. in 1993 [27]. An X-ray-guided catheter is introduced through the femoral vein to the varicose vein. Subsequently, an embolization spiral is inserted into the dilated area, which closes the pathological vessel. Prospective studies carried out by Kim et al., demonstrate that during the 48 -month observation period the procedure 
was effective in $83 \%$ of cases. No beneficial effects were found in $13 \%$ of patients, whereas deterioration of symptoms was observed in $4 \%$ of cases. There were no side-effects, such as abnormal menstruation or hormonal imbalance. Moreover, the embolisation procedure did not reduce fertility in the examined group. [21, 28].

The aetiology of CPP is complex and therefore multidirectional diagnostic procedures are required. The first step involves history taking and physical examination. An important element of history taking is to determine the factor inducing or increasing pain, and to assess the effects of pain on the quality of life as well as the way of coping with it. The diagnosis of CPP also includes imaging examinations, i.e. transabdominal and transvaginal ultrasound of the reproductive system and pelvis, computer tomography (CT), magnetic resonance imaging (MRI) and venography. Ultrasound is used to exclude other organic changes that could induce CPP, i.e. ovarian pathology, uterine fibroids, adenomyosis or dilated vessels of adnexa. The use of colour Doppler ultrasound enables visualisation of increased flow in the parametrial vessels. Park et al. have determined the diagnostic CPP criteria for transvaginal and transabdominal ultrasound examinations [7, 20, 21, 22], which include:

- tortuous veins of the pelvis of a diameter $>6 \mathrm{~mm}$

- slowed down or retrograde vascular flow

- dilated, arched vein within the myometrium communicating bilaterally with pelvic varices

- polycystic image of ovaries.

The diagnostic procedures for CPP also include biochemical blood tests, bacteriological tests, cystoscopy, and contrast examinations of the large intestine. Diagnostic laparoscopy is performed in $40 \%$ of CPP cases. The procedure enables finding the foci of endometriosis in $1 / 3$ of cases and adhesions in $1 / 4$ of patients; however, in $1 / 3$ of cases it does not visualise the possible cause [29].

Based on a meta-analysis by Latthe et al., the risk factors of chronic pelvic pain have been determined. They include: dysmenorrhoea in women $<30$ years of age, Body Mass Index $(\mathrm{BMI})<20$, smoking, menarche at the age $<12$ years, long menstruation cycles, prolonged and irregular menstrual bleedings, symptoms of premenstrual syndrome, infertility, history of sexual abuse, mental disorders (e.g. depression, anxiety, hysteria) [30, 31].

The treatment of CPP is multi-directional. The first stage algorithms involve pharmacological therapy. Once ineffective, interventional procedures are instituted. The pharmacological agents minimising pain include analgesics (NSAIDs, opioids), antibiotics, contraceptive pills, progestagens, GnRH agonists, danazol. According to the RCOG, hormonal therapy should be used for 3-6 months. Reginald et al., who used dihydroergotamine in patients with PCS, observed reduced passive congestion and alleviated pain sensations. The administration of $30 \mathrm{mg}$ of medroxyprogesterone acetate (MPA) daily decreased the features of congestion within the pelvis observed on venography and reduced the severity of pain. The alternative method of PCS treatment is ovarian and internal iliac vein embolization. Surgical procedures used to reduce CPP depend on the likely cause of complaints and include the following: removal or vaporisation of the vulval or vestibular abnormal tissue, caused by the inflammation of vestibular glands or vulvodynia, hysteroscopic removal of lesions visualised in the uterine cavity, myomectomy, lysis of intraperitoneal adhesions, removal of endometriosis foci, disruption of conduction pathways - presacral neurectomy (PSN), laparoscopic uterine nerve ablation (LUNA) in cases of dysmenorrhoea, adhesions, in the course of endometriosis, adenomyosis, CPP of indiscernible causes, appendectomy, surgical hernia ring closure, and trigger point therapy (local anaesthesia) [7, 24-26].

Data available in the Cochrane library compare the pharmacological and non-pharmacological methods used to relieve chronic pelvic pain. The extent of pain reduction, quality of life, adverse side-effects and economic benefits were evaluated in a randomised study. Several groups were distinguished in which the following were used: changes in lifestyle, psychotherapy, physiotherapy, pharmacotherapy (non-steroidal anti-inflammatory drugs, DTA, progesterone, IUD, Danazol, GnRH analogues, drugs affecting venous vessels, antidepressants, antiepileptic drugs, analgesics), surgical interventions (laparoscopy, hysterectomy, oophorectomy).

The exclusion criteria were endometriosis, irritable bowel syndrome, primary dysmenorrhoea, pain syndrome associated with chronic inflammation. Reduced pain was observed in the groups treated with MDA and goserelin. Otherwise, the liberation of adhesions, sertraline and laparoscopic disruption of conduction pathways did not result in pain elimination $[25,26,32,33]$.

\section{CONCLUSIONS}

Due to the complex aetiology of chronic pelvic pain, the most beneficial effects are obtained when the therapy is based on cooperation between the gynaecologist, physiotherapist, psychologist and interventional radiologist.

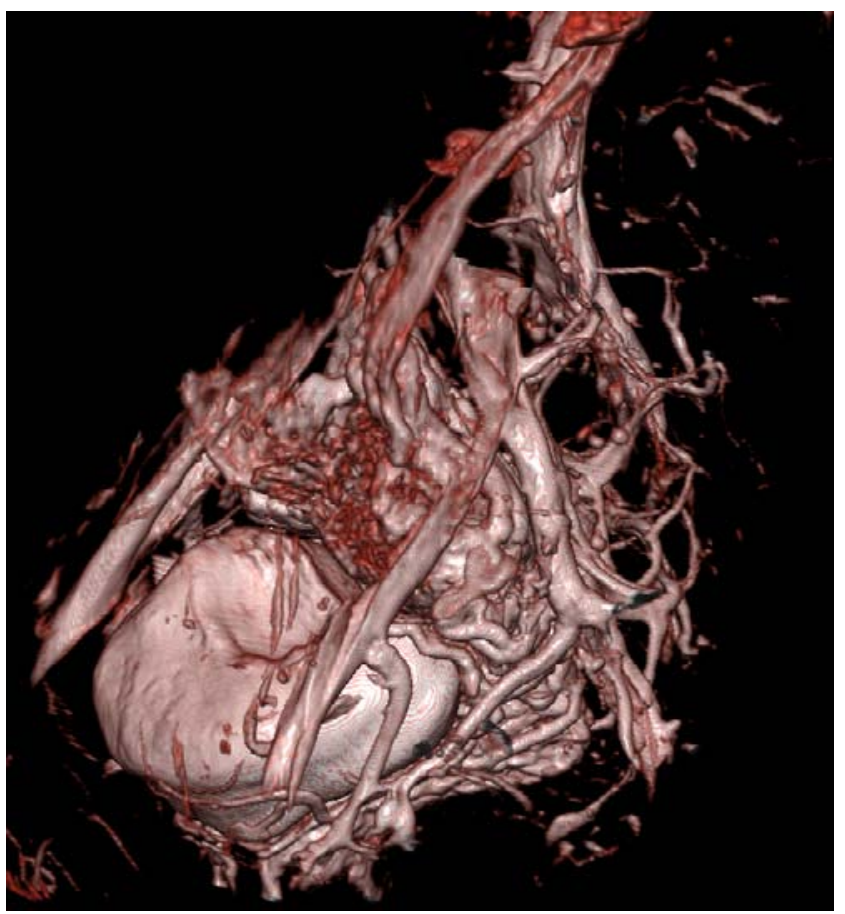

Figure 1. Magnetic resonance scan showing enhanced, bilateral tortuous pelvic veins 

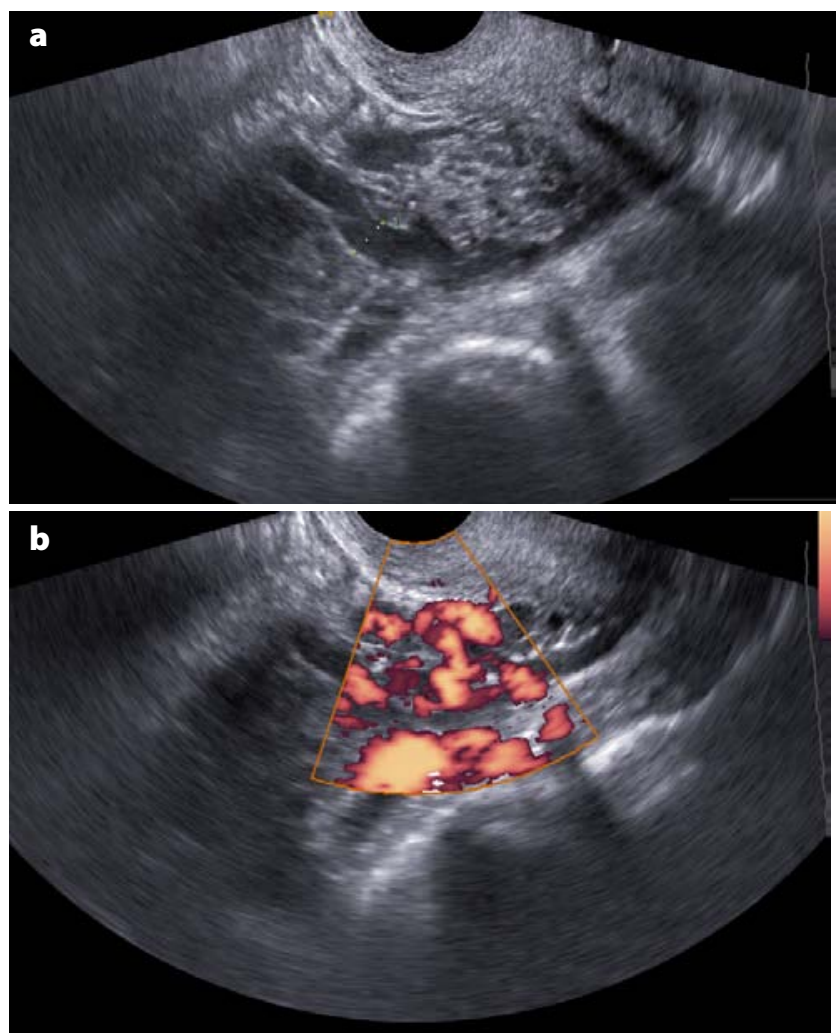

Figure 2. Gray-scale (a) and colour Doppler (b) ultrasound images demonstrate congested pelvic veins

\section{REFERENCES}

1. Zondervan KT, Yudkin PL, Vessey MP. The community prevalence of chronic pelvic pain in women and associated illness behaviour. Br J Gen Pract. 2001; 51: 541-7.

2. Triolo $\mathrm{O}$, Laganà $\mathrm{AS}$, Sturlese E. Chronic pelvic pain in endometriosis: an overview. J Clin Med Res. 2013; 5: 153-63.

3. Perry CP. Current concepts of pelvic congestion and chronic pelvic pain. JSLS. 2001; 5: 105-110.

4. Gelbaya TA, El-Halwagy HE. Focus on primary care: chronic pelvic pain in women. Obstet Gynecol Surv. 2001; 56: 757-764.

5. Malykhina AP. Neural mechanisms of pelvic organ cross-sensitization. Neuroscience. 2007; 149: 660-672.

6. Wesselmann U. Neurogenic inflammation and chronic pelvic pain. World J Urol. 2001; 19: 180-185.

7. Royal College of Obstetricians and Gynaecologists: Development of RCOG Green-top Guidelines: The initial management of chronic pelvic pain. No. 41. London: RCOG; 2012.

8. Neis KJ, Neis F. Chronic pelvic pain: cause, diagnosis and therapy from a gynaecologist's and an endoscopist's point of view. Gynecol Endocrinol. 2009; 25: 757-761.

9. Revised American Society for Reproductive Medicine classification of endometriosis: 1996. Fertil Steril. 1997; 67: 817-821.

10. Randall GW, Gantt PA, Poe-Zeigler RL, [et al.]. Serum antiendometrial antibodies and diagnosis of endometriosis. Am J Reprod Immunol. 2007; 58: 374- 382 .
11. Berkley KJ, Rapkin AJ, Papka RE. The pains of endometriosis. Science. 2005; 308: 1587-1589.

12. Raab S, Plate KH. Different networks, common growth factors: shared growth factors and receptors of the vascular and the nervous system. Acta Neuropathol. 2007; 113: 607-626.

13. Bedaiwy MA, Falcone T, Goldberg JM. Peritoneal fluid leptin is associated with chronic pelvic pain but not infertility in endometriosis patients. Hum Reprod. 2006; 21: 788-791.

14. Laganà AS, Salmeri FM, Retto G. Stage-related changes of peritoneal soluble TNF $\alpha$ and TNFR1 and TNFR2 in cells recovered from peritoneal fluid of women with endometriosis. J Reprod Immunol. 2012; 94: 94-95.

15. Dawood MY, Khan-Dawood FS, Wilson L. Peritoneal fluid prostaglandins and prostanoids in women with endometriosis, chronic pelvic inflammatory disease, and pelvic pain. Am J Obstet Gynecol. 1984; 148: 391-395.

16. Drosdzol-Cop A, Skrzypulec-Plinta V, Stojko R. Serum and peritoneal fluid immunological markers in adolescent girls with chronic pelvic pain. Obstet Gynecol Surv. 2012; 67: 374-381.

17. Taylor HC Jr. Vascular congestion and hyperemia; their effect on function in the female reproductive organs; clinical aspects of the congestion fibrosis syndrome. Am J Obstet Gynecol. 1949; 57: 637-653.

18. Metzger DA. Pelvic congestion. In: Steege JF, Metzger DA, Levy BS. Chronic Pelvic Pain: An Integrated Approach. Philadelphia: WB Saunders Co, 1999, 149-160.

19. Kaufman JA. Inferior vena cava and tributaries. In: Kaufman JA, Michael JL, eds. The Requisites: Vascular and Interventional Radiology. Philadelphia, PA: Mosby, 2004, 273-273.

20. Perry CP. Current concepts of pelvic congestion and chronic pelvic pain. JSLS. 2001; 5: 105-110.

21. Freedman J, Ganeshan A, Crowe PM. Pelvic congestion syndrome: the role of interventional radiology in the treatment of chronic pelvic pain. Postgrad Med J. 2010; 86: 704-710.

22. Park SJ, Lim JW, Ko YT. Diagnosis of pelvic congestion syndrome using transabdominal and transvaginal sonography. AJR Am J Roentgenol. 2004; 182: 683-688.

23. Rhodin A. Successful management of chronic pelvic pain. J Pain Palliat Care Pharmacother. 2013; 27: 289-291.

24. Jarrell JF, Vilos GA, Allaire C. Chronic Pelvic Pain Working Group; SOGC Consensus Guidelines for the Management of Chronic Pelvic Pain. J Obstet Gynaecol Can. 2005; 27: 781-801.

25. Brown J, Kives S, Akhtar M. Progestagens and anti-progestagens for pain associated with endometriosis. Cochrane Database of Systematic Reviews 2012; (3): CD002122.

26. Selak V, Farquhar C, Prentice A, Singla A. Danazol for pelvic pain associated with endometriosis. Cochrane Database Syst Rev. 2007: (4): CD000068.

27. Edwards RD, Robertson IR, MacLean AB, [et al.]. Case report: pelvic pain syndrome successful treatment of a case by ovarian veinembolisation. Clin Radiol. 1993; 47: 429-431.

28. Kim HS, Malhotra AD, Rowe PC. Embolotherapy for pelvic congestion syndrome: long-term results. J Vasc Interv Radiol. 2006; 17: 289-297.

29. Howard FM. Chronic pelvic pain in women. Am J Manag Care. 2001; 7: 1001-111.

30. Latthe P, Latthe M, Say L, Gulmezoglu M, Khan KS. WHO systematic review of prevalence of chronic pelvic pain: a neglected reproductive health morbidity. BMC Public Health 2006; 6: 177.

31. Latthe P, Mignini L, Gray R, Hills RK, Khan KS. Factors predisposing women to chronic pelvic pain: systematic review. BMJ 2006; 332: 749-55.

32. Cheong YC, Smotra G, Farquhar C. Non surgical interventions for the management of chronic pelvic pain. Cochrane Database of Systematic Reviews 2010; (11) CD008797.

33. Stones W, Cheong YC, Howard FM, Singh S. Interventions for treating chronic pelvic pain in women. Cochrane Database of Systematic Reviews 2005; (2) CD000387. 